\title{
Educación quirúrgica durante la pandemia de COVID-19: primer consenso nacional de la División de Educación de la Asociación Colombiana de Cirugía
}

\author{
Surgical education during the COVID-19 pandemic: first national \\ consensus of the Education Division of the Colombian Surgery Association
}

\author{
Luis Mauricio Figueroa-Gutiérrez $\mathbb{D}$
}

Médico, especialista en Cirugía general y Cirugía pediátrica, profesor asistente de Cirugía Pediátrica, Universidad del Valle, Cali, Colombia.

He leído con interés el artículo "Educación quirúrgica durante la pandemia de COVID-I9: primer consenso nacional de la División de Educación de la Asociación Colombiana de Cirugía" ', publicado en el último número de la Revista Colombiana de Cirugía. En primer lugar se debe resaltar el papel protagónico que la División de Educación de la Asociación Colombiana de Cirugía y el Comité editorial de la Revista han tenido durante todo el desarrollo de la pandemia, lo que ha permito la elaboración y publicación de diversos documentos y recomendaciones encaminadas a que, desde las diferentes especialidades de la cirugía, se tengan instrumentos de trabajo basados en la mejor evidencia disponible sobre esta nueva condición clínica, que permitan dar respuesta a las necesidades de los pacientes, velando por la protección del personal de la salud ${ }^{2,3}$.
Con respecto a los nueve puntos definidos como recomendaciones para los programas de especializacion en cirugía general, todos son de importancia capital y podrían ser aplicables a cualquier programa de especialización médico-quirúrgica en el país, pero me llama la atención que, tal vez de manera involuntaria, se haya omitido un aparte sobre el papel de la telesalud como herramienta útil, tanto para la asistencia, como para la docencia ${ }^{4,5}$. Se comenta, en el punto seis, sobre la importancia de la educación utilizando las herramientas virtuales existentes en la actualidad para la revisón de casos, clubes de revistas, revisiones de tema y junta de decisiones (esto considerado en la normatividad actual como telexperticia) ${ }^{6}$, pero no se profundiza en lo que desde ya hace más de una década se viene incorporando, como un recurso válido en diferentes especialidades quirúrgicas ${ }^{7,8,9}$.

Palabras clave: COVID I9; infecciones por coronavirus; pandemias; cirugía general; educación médica; programas de postgrado.

Keywords: COVID I9; coronavirus infections; pandemics; general surgery; education, medical; health postgraduate programs.

Fecha de recibido: 1/08/2020 - Fecha aceptación: 4/08/2020

Correspondencia: Luis Mauricio Figueroa-Gutiérrez, Carrera 19 \# 94-96, Villasol Manzana B Casa 19, Pereira, Colombia.

Teléfono 3137371166

Correo electrónico: Imfg13@yahoo.es

Citar como: Figueroa-Gutiérrez LM. Educación quirúrgica durante la pandemia de COVID-19: primer consenso nacional de la División de Educación de la Asociación Colombiana de Cirugía. Rev Colomb Cir. 2020;35:705-7. https://doi.org/10.30944/20117582.739

Este es un artículo de acceso abierto bajo una Licencia Creative Commons - BY-NC-ND https://creativecommons.org/licenses/by-ncnd/4.0/deed.es 
Se plantea, en el punto cuatro, que el volumen operatorio de los residentes ha venido en descenso por las restricciones en cuanto a la programación de procedimientos electivos, pero considerando que la enseñanza durante una residencia quirúrgica no sólo involucra los servicios de hospitalización, urgencias y las salas de operaciones, y las prácticas que allí se llevan a cabo, sino que los escenarios de consulta de primera vez, las de seguimiento y los controles postoperatorios, son áreas en las que también se adquieren habilidades, y que se han visto afectadas por la pandemia, pero no se plantean recomendaciones con el fin de superar estas adversidades.

En este sentido, existiendo múltiples experiencias exitosas en telesalud en el país ${ }^{\mathrm{IO}, \mathrm{II}}$, se debería recomendar a los programas, fomentar en las instituciones la educación a los docentes y residentes en comunicación virtual efectiva o "webside manner", y en el conocimiento de las herramientas que vienen en desarrollo a partir de las exigencias actuales, como por ejemplo Trello (software de administración de proyectos, que integra ideas, tareas e imágenes), que permite la programación y la gestión de un programa de teleconsulta ${ }^{\text {II }}$ y PROXIMIE ${ }^{\circ}$, plataforma que permite a los cirujanos interactuar y compartir conocimientos de forma remota (telementorización), utilizando un móvil, un computador personal portátil o una tableta ${ }^{12}$.

Los lineamientos expedidos por el Estado para enfrentar la pandemia han flexibilizado e incrementado las ofertas de telesalud en Colombia, permitiendo que las actividades de telemedicina interactiva y no interactiva, la telexperticia, la teleorientación y el teleapoyo ${ }^{\mathrm{I}}$, puedan ser practicados por los docentes y enseñados a los residentes, para que aprendan sobre estas nuevas estrategias de atención que, como queda claro en las experiencias publicadas recientemente ${ }^{14,15,16}$, no pretenden reemplazar la atención quirúrgica usual, pero para estos momentos pueden ser de utilidad para disminuir el desplazamiento de los pacientes y sus cuidadores, acercar las especialidades a las áreas rurales, y disminuir los costos y el tiempo de movilización de los enfermos ${ }^{17}$.
Ya existen evidencias en el mundo en el campo de los controles postquirúrgicos ${ }^{18} \mathrm{y}$ en programas de cuidado de heridas, que están utilizando estos recursos con anterioridad ${ }^{19}$.

Con respecto a la simulación y la telementorización, en cirugía pediátrica se han presentado estrategias como las del Hospital Italiano de Buenos Aires en su Ateneo Bibliográfico denominado Fundamentals of Laparoscopic Surgery simulator training (https://www.youtube.com/ watch? $\mathrm{v}=\mathrm{mDTaRs92 \textrm {s } 7 0}$ ), para que residentes $\mathrm{y}$ profesionales en su casa puedan continuar con el entrenamiento en equipos elaborados con materiales de bajo costo, mientras no se les permita asistir a los laboratorios de las universidades, así como también estrategias de enseñanza a estudiantes de pregrado, para el aprendizaje de procedimiento básicos, guiados a distancia; todo lo cual demuestra que la innovación en la pandemia nos lleva a diseñar soluciones que pueden llegar a superar las propuestas existentes, que son de alto costo ${ }^{20}$.

Para finalizar, a partir de los caminos trazados en telemedicina por Willem Einthoven en $1906^{21}$, la pandemia COVID-I9 nos exige que aprovechemos los desarrollos tecnológicos con que contamos en la actualidad, involucremos las diversas estancias de las instituciones de educación superior, las Entidades Prestadoras de los Servicios de Salud (EPS) ${ }^{\text {II }} \mathrm{y}$ los entes gubernamentales, para que aprovechando las Tecnologías de la Información y la Comunicación (TICs), una vez superada la pandemia, la telesalud, con todos sus componentes, permita mejorar, tanto la asistencia a los pacientes, como la educación en salud, en todos los niveles de enseñanza.

\section{Referencias}

I. Domínguez-Torres LC, Torregrosa-Almonacid L, Sánchez-Maldonado W, Lasprilla N, Vargas-Barato F, Niño-Rodríguez ÁE, Rosero-Rosero GA, et al. Educación quirúrgica durante la pandemia COVID-I9: Primer consenso nacional de la División de Educación de la Asociación Colombiana de Cirugía. Rev Colomb Cir. 2020;35:363-72. https://doi.org/I0.30944/20I17582.685

2. Cuevas-López L, Ayala-Acosta JC, Velásquez-Jiménez OA, Navarro-Alean JA, González-Higuera LG, Zuri- 
ta-Medrano N, et al. Recomendaciones para el manejo de los pacientes quirúrgicos urgentes durante la pandemia COVID-I9. Rev Colomb Cir. 2020;35:I43-52. https://doi.org/I0.30944/20117582.619

3. Torregrosa-Almonacid L, Prieto-Ortiz RG, Cabrera-Vargas LF, Ordoñez-Mosquera JM, Sánchez-Cortés EF, Rodríguez-Vargas CM, et al. Recomendaciones generales para los Servicios de Cirugía en Colombia durante la pandemia COVID-I9 (SARS-CoV-2). Rev Colomb Cir. 2020;35:264-8o. https://doi.org/I0.30944/20II7582.633

4. Huang EY, Knight S, Guetter CR, Davis CH, Moller $\mathrm{M}$, Slama E, et al. Telemedicine and telementoring in the surgical specialties: A narrative review. Am J Surg. 2019;2I8:760-6.

https://doi.org/IO.IoI6/j.amjsurg.2019.07.018

5. Figueroa LM. Telesalud en Colombia, retos asociados con el COVID-I9. Biomédica. 2020;40(Supl. 2). Publicación anticipada. https://doi.org/I0.7705/biomedica.5594

6. Ministerio de Salud y Protección Social. Resolución 2654 de 2019. Fecha de consulta: 5 de mayo de 2020. Disponible en: https://www.minsalud.gov.co/Normatividad_Nuevo/Resoluci\%C3\%B3n\%20No.\%202654\%20 del\%2020I9.pdf

7. Eadie LH, Seifalian AM, Davidson BR. Telemedicine in surgery. Br J Surg. 2003;90:647-58. https://doi.org/IO.IOO2/bjs.4I68

8. Asiri A, AlBishi S, AlMadani W, ElMetwally A, Househ M. The use of telemedicine in surgical care: A systematic review. Acta Inform Med. 20I8;26:20I-6. https://doi.org/IO.5455/aim.2018.26.20I-206

9. Marescaux J, Leroy J, Gagner M, Rubino F, Mutter D, Vix M, et al. Transatlantic robot-assisted telesurgery. Nature. 200I;4I3:379-80. https://doi.org/IO.I038/35096636

Io. Rey-Moreno C, Reigadas JS, Villaba EE, Vinagre JJ, Fernández AM. A systematic review of telemedicine projects in Colombia. J Telemed Telecare. 2010;16:I14-9. http://doi.org/IO.I258/jtt.2009.090709

II. Márquez-Velásquez JR. Teleconsulta en la pandemia por Coronavirus: desafíos para la telemedicina pos-COVID-I9. Rev Col Gastroenterol. 2020;35(Suppl I):5-I6. http://dx.doi.org/I0.225I6/25007440.543

I2. Rodríguez-García JI, Contreras-Sáiz E, García-Munar M, García-Flórez L, Granero-Trancón J. Telemedicina, telementorización y evaluación telemática en cirugía.
¿Es su momento después de la COVID-r9?. Cir Esp. 2020;Sooo9-739X(20)30193-7. Publicación anticipada. http://doi.org/IO.IOI6/j.ciresp.2020.05.022

I3. Ministerio de Salud y Protección Social. Telesalud y telemedicina para la prestación de servicios de salud en la pandemia por COVID-19. Abril 2020. Fecha de consulta: 3I de julio de 2020. Disponible en: https:// www.minsalud.gov.co/Ministerio/Institucional/Procesos\%20y\%2oprocedimientos/PSSSo4.pdf

I4. Contreras CM, Metzger GA, Beane JD, Dedhia PH, Ejaz A, Pawlik TM. Telemedicine: Patient-provider clinical engagement during the COVID-I9 pandemic and beyond. J Gastrointest Surg. 2020;24:I692-7. http://doi.org/I0.I007/sir605-020-04623-5

I5. Hakim AA, Kellish AS, Atabek U, Spitz FR, Hong YK. Implications for the use of telehealth in surgical patients during the COVID-I9 pandemic. Am J Surg. 2020;220:48-9.

http://doi.org/Io.IOI6/j.amjsurg.2020.04.026

I6. Gadzinski AJ, Ellimoottil C. Telehealth in urology after the COVID-I9 pandemic. Nat Rev Urol. 2020;17:363-4. https://doi.org/I0.I038/s4I585-020-0336-6

I7. James HE. Pediatric neurosurgery telemedicine clinics: a model to provide care to geographically underserved areas of the United States and its territories. J Neurosurg Pediatr. 2016;25:753-7. http://doi.org/I0.3I7I/20I6.6.PEDSI6202

I8. Gunter RL, Chouinard S, Fernandes-Taylor S, Wiseman JT, Clarkson S, Bennett K, et al. Current use of telemedicine for post-discharge surgical care: A systematic review. J Am Coll Surg. 20I6;222:915-27. http://doi.org/IO.IoI6/j.jamcollsurg.2016.0I.062

19. Sood A, Granick MS, Trial C, Lano J, Palmier S, Ribal E, et al. The role of telemedicine in wound care: A review and analysis of a database of 5,795 patients from a mobile wound-healing center in Languedoc-Roussillon, France. Plast Reconstr Surg. 2016;138(3 Suppl):248S-56S. http://doi.org/Io.I097/PRS.0000000000002702

20. Bogen EM, Augestad KM, Patel HR, Lindsetmo RO. Telementoring in education of laparoscopic surgeons: An emerging technology. World J Gastrointest Endosc. 20I4;6:I48-55. http://doi.org/IO.4253/wjge.v6.i5.I48

2I. Strehle EM, Shabde N. One hundred years of telemedicine: does this new technology have a place in paediatrics? Arch Dis Child. 2006;91:956-9. http://doi.org/IO.II36/adc.2006.099622 